\title{
3D Modeling of Outdoor Environments by Integrating Omnidirectional Range and Color Images
}

\author{
Toshihiro ASAI, Masayuki KANBARA, Naokazu YOKOYA \\ Nara Institute of Science and Technology \\ 8916-5 Takayama Ikoma Nara, Japan 630-0192 \\ \{toshih-a, kanbara, yokoya\}@is.naist.jp
}

\begin{abstract}
This paper describes a 3D modeling method for wide area outdoor environments which is based on integrating omnidirectional range and color images. In the proposed method, outdoor scenes can be efficiently digitized by an omnidirectional laser rangefinder which can obtain a 3D shape with high-accuracy and by an omnidirectional multi-camera system (OMS) which can capture a high-resolution color image. Multiple range images are registered by minimizing the distances between corresponding points in the different range images. In order to register multiple range images stably, points on plane portions detected from the range data are used in registration process. The position and orientation acquired by RTK-GPS and gyroscope are used as initial values of simultaneous registration. The $3 D$ model obtained by registration of range data is mapped by textures selected from omnidirectional images in consideration of the resolution of texture and occlusions of the model. In experiments, we have carried out $3 D$ modeling of our campus with the proposed method.
\end{abstract}

\section{Introduction}

3D models of outdoor environments can be used in a number of fields such as site simulation, human navigation and virtual walk-through. However, such 3D models are often made manually with high costs, so recently automatic 3D modeling methods have been widely investigated; for example, 3D shape estimation from an image sequence [1]-[5] and 3D measurement of outdoor environments by a laser rangefinder [6]-[10]. The merit of the former is that the method can reconstruct a 3D model with only a camera. However, since it is difficult for vision-based methods to reconstruct a 3D model with high accuracy, the approaches are not suitable for 3D modeling of wide area outdoor environments. On the other hand, the latter can widely measure the shape of object with high accuracy. Thus it is useful for wide area outdoor environments.
This paper describes a 3D reconstruction method for wide area outdoor environments. In this paper, in order to measure wide outdoor environments, we use an omnidirectional laser rangefinder which can measure the distances from the sensor to objects with high accuracy and an omnidirectional multi-camera system (OMS) which can capture a wide-angle high-resolution image. Using the two omnidirectional sensors, wide outdoor environments can be digitized efficiently. Note that omnidirectional range and color images are acquired approximately at the same position to register both of the images geometrically.

One of the most important problems in modeling using a rangefinder is registration of multiple range data. In most conventional methods for registration of multiple range data, the ICP algorithm [15] is often used. In the method, the distance between corresponding points in paired range data is defined as registration error, and a transformation matrix is calculated so that the error is minimized. Therefore, to apply the ICP algorithm, it is necessary for a pair of range data to include overlapped regions. Since outdoor environments are complex, many occlusions occur and there are objects which cannot be measured by the rangefinder stably. In order to solve this problem, Gunadi et al. [11] register local range data acquired on the ground into a global range data acquired from the sky. Zhao et al. [8] and Früh et al. [12] acquire the range data and estimate the position and orientation of the rangefinder while it is moving. In these methods, the precision of generated model is determined by the precision of sensor position and orientation. These methods need to measure the sensor position and orientation under a motion, accurately.

In this paper, in order to register multiple range data of wide area stably, only planar surfaces that are extracted from the range data are used in registration process because typical outdoor environments contain many plane regions such as walls and roads. Using the detected plane portions, the range data are simultaneously registered by the improved ICP algorithm [16]'[17] . In this case, the position and orientation of sensor system, which are measured 


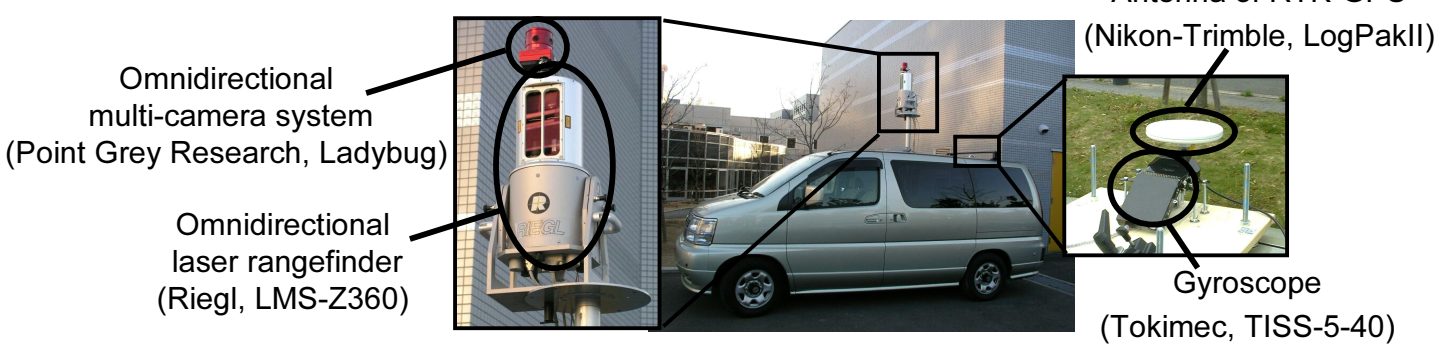

Figure 1. Sensor system mounted on a vehicle.

by RTK-GPS and gyroscope, are used as initial values in optimization by the ICP algorithm. The surface model is generated by polygonizing the registered range data. The surface model is then texture-mapped by omnidirectional images selected in consideration of resolution and occlusion.

This paper is structured as follow. Section 2 briefly describes the sensor system used in this study. Section 3 explains the registration of range data acquired at multiple positions. Section 4 describes the method for generating a textured model mapped by selected omnidirectional images. In Section 5, experimental results of wide area 3D modeling are described. Finally, Section 6 gives summary and future work.

\section{Sensor System}

This section describes the sensor system. Fig. 1 illustrates the sensor system mounted on a vehicle. In the proposed method, the position and orientation of sensor system are fixed during acquisition of data. The system equips the omnidirectional laser rangefinder (Riegl, LMS-Z360), omnidirectional multi-camera system (Point Grey Research, Ladybug), RTK-GPS (Nikon-Trimble, LogPakII), and gyroscope (Tokimec, TISS-5-40).

\subsection{Specifications of Sensors}

\section{Omnidirectional Rangefinder}

The specification of the rangefinder is shown in Table 1. Angle and resolution of measured range data can be determined by user. Fig. 2 shows an example of acquired omnidirectional range image in which the distance from the rangefinder is coded in intensity. This rangefinder measures environments omnidirectionally by illuminating a laser beam radially. Therefore, the density of measuring points becomes sparse in a far place and dense in a near place from the sensor. The rangefinder takes at least $45 \mathrm{sec}-$ onds for omnidirectional measurement.
Table 1. Specification of LMS-Z360

\begin{tabular}{|c|c|}
\hline measurable angle & $\begin{array}{cc}\text { horizontal: } & 360^{\circ} \\
\text { vertical: } & -50^{\circ} \sim 40^{\circ}\end{array}$ \\
\hline measurable range & $1 \mathrm{~m} \sim 200 \mathrm{~m}$ \\
\hline measurement accuracy & $\pm 12 \mathrm{~mm}$ \\
\hline minimum step angle & $0.01^{\circ}$ \\
\hline maximum resolution & $\begin{array}{cc}\text { horizontal: } & 0.0025^{\circ} \\
\text { vertical: } & 0.002^{\circ}\end{array}$ \\
\hline
\end{tabular}

\section{Omnidirectional Multi-camera System (OMS)}

Fig. 3 shows an example of omnidirectional color image generated from images acquired by the OMS Ladybug. The OMS has radially located six camera units in a camera block and their positions and orientations are fixed. Since each camera can acquire a $768 \times 1024$ pixels image, the OMS can acquire high-resolution omnidirectional color images which covers more than $75 \%$ of full spherical view. The Ladybug is calibrated geometrically and photometrically in advance [14].

\section{RTK-GPS and gyroscope}

RTK-GPS and gyroscope are used to measure the position and orientation of sensor system, respectively. The specification of RTK-GPS is shown in Table 2 (a). In general, a yaw value measured by the gyroscope includes an accumulative error. The gyroscope is interlocked with RTKGPS in order to correct the accumulative error by measuring the direction of movement calculated by GPS data under motion. The specification of gyroscope is shown in Table 2 (b).

\subsection{Alignment of Sensor Coordinate Systems}

There are four coordinate systems in the sensor system; the rangefinder, OMS, RTK-GPS, and gyroscope coordinate systems. Geometrical relationship among the coordinate systems is fixed, and these coordinate systems are regis- 


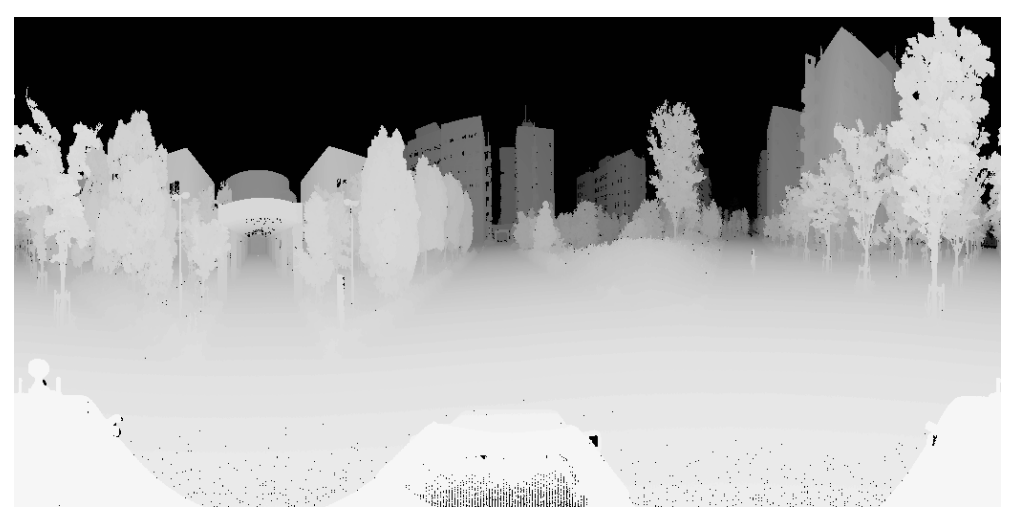

Figure 2. Omnidirectional range image.

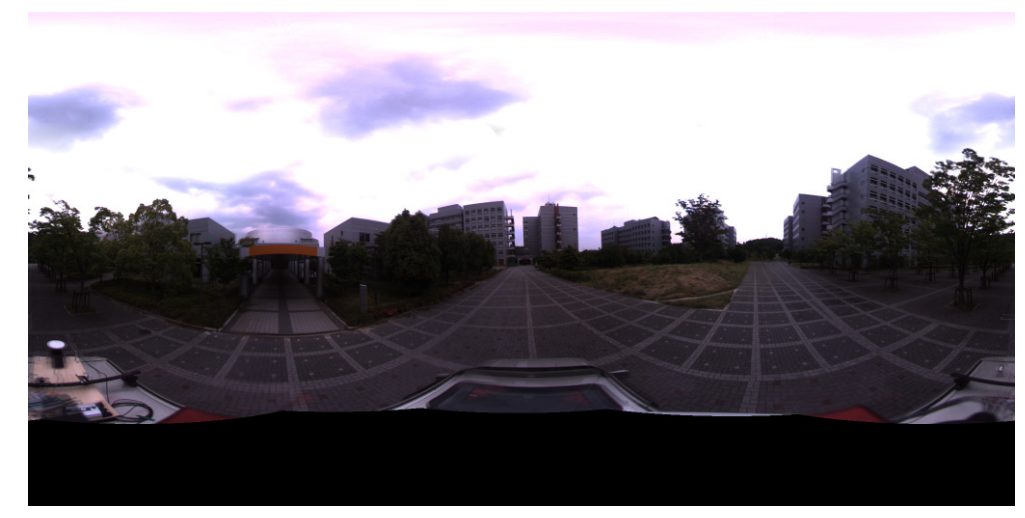

Figure 3. Omnidirectional color image.

\section{Table 2. Specifications of RTK-GPS and gyro- scope}

(a) Accuracy of RTK-GPS.

\begin{tabular}{|c|c|}
\hline latitude & $\pm 3.0 \mathrm{~cm}$ \\
\hline longitude & $\pm 3.0 \mathrm{~cm}$ \\
\hline altitude & $\pm 4.0 \mathrm{~cm}$ \\
\hline
\end{tabular}

(b) Accuracy of gyroscope.

\begin{tabular}{|c|c|}
\hline yaw & $\pm 2^{\circ}$ \\
\hline roll & $\pm 0.5^{\circ}$ \\
\hline pitch & $\pm 0.5^{\circ}$ \\
\hline
\end{tabular}

tered to RTK-GPS (global) coordinate system as shown in Fig. 4. Figs. 4(a), (b) and (c) illustrate transformation matrices among sensor coordinate systems. The methods for estimating the transformation matrices are described below. (a) Transformation between OMS and rangefinder coordinate systems : By giving corresponding points in range and color images, the transformation matrix can be estimated by the method in [14].

(b) Transformation between rangefinder and gyroscope coordinate systems : The transformation matrix can be estimated by measuring more than three markers whose po- sitions in the gyroscope coordinate system are known. The markers are placed at positions which can be measured by the rangefinder as shown in Fig. 5(a). The transformation (b) in Fig. 4 is estimated by measuring the position of markers with respect to the rangefinder as shown in Fig. 5(b).

(c) Transformation between gyroscope and global coordinate systems : The coordinate systems of RTK-GPS (global coordinate system) and gyroscope are aligned automatically, because these sensors are interlocked with each other.

\section{Generation of 3D Surface Model}

A surface model of environment is generated by integrating multiple range data. Therefore, all range data are first registered to the GPS coordinate system. Since the position and orientation acquired by the sensors include some errors, they are used as initial values in registration and should be optimized. The ICP algorithm [15] is usually used for optimal registration of multiple range images. In the conventional ICP algorithm, the distance between corresponding 


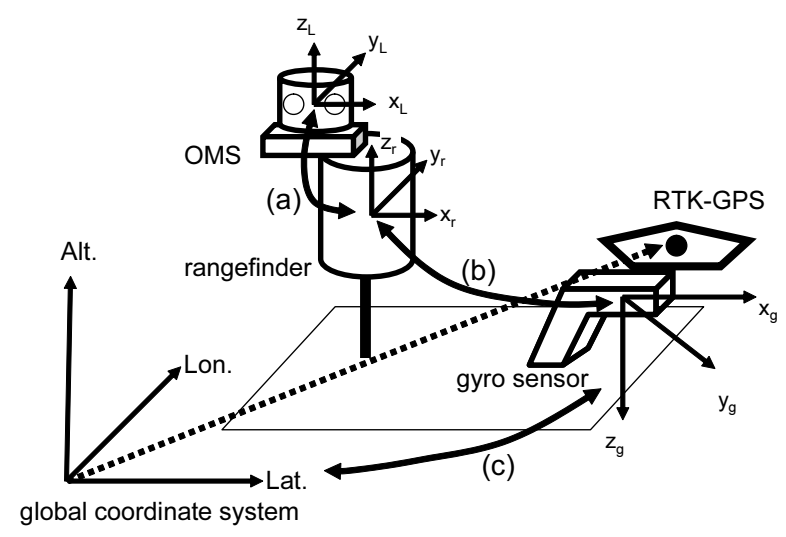

Figure 4. Relationship among the coordinate systems of the sensors.

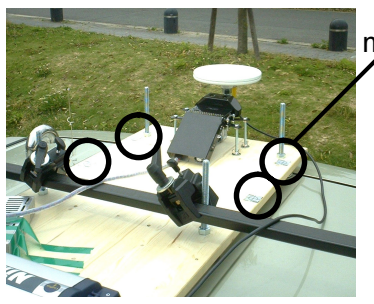

(a) Layout of markers

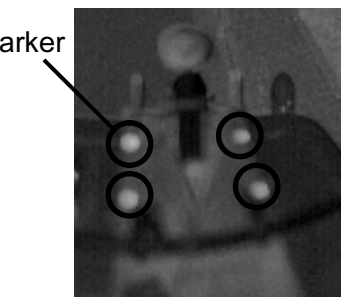

(b) Range image of markers
Figure 5. Alignment of rangefinder and gyroscope coordinate systems.

points in paired range data is defined as a registration error, and the transformation matrix is calculated so that the error is minimized. The present rangefinder measures the distance by rotating the laser scan, thus the spatial density of data points depends on the distance; that is, close objects are measured densely and far objects are measured sparsely. In registering range data obtained at different positions, this causes the problem that multiple points may correspond to a single point. The solution tends to fall into a local minimum, because the correspondences between points are discrete and do not include the surface information about an object [16]. The proposed method first detects plane regions from all range data, and then determines point-to-plane correspondences. Finally, the transformation matrix of range data is calculated by overlapping the corresponding planes.

\subsection{Simultaneous Registration of Multiple Range Data}

Multiple range data are registered by overlapping the corresponding planes among range data. For this purpose as a pre-processing, planar regions in range data are detected and the normal vectors at the measured points in pla- nar regions are calculated, and then the plane in one data is searched which corresponds to each point in the other data. Multiple range data are simultaneously registered for optimization of transformation matrices. The position and orientation acquired by RTK-GPS and gyroscope are used as an initial value in the optimization. the flowchart of the registration is shown in Fig. 6 and the detail of each process is described in the following.

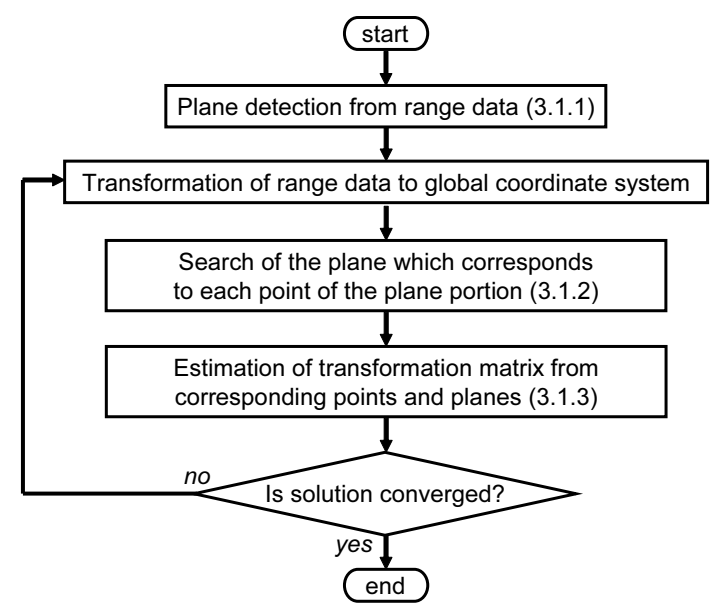

Figure 6. Procedure of registring range data

3.1.1. Plane Detection from Range Data. Planar regions are detected from range data by local plane fitting. We employ the renormalization method [13] for planar region detection and the quadtree segmentation recursively. The whole range image is taken as an initial region. The distances between estimated plane and points in the region are calculated and when at least one distance is bigger than a threshold, the region is split. On the other hand, when all the distances are smaller than the threshold, the region is defined as a plane portion. The points which are not defined as a plane portion are not used for registration process.

3.1.2. Search of Corresponding Plane. The plane portion of range data is searched from different range data. The plane correspondence is described below. Let $R D_{n}$ be range data $n(n=1, \cdots, N), P_{n i}$ be a planar region in $R D_{n}(i=1, \cdots, I)$ and $Q_{n i j}$ be a point in the plane $P_{n i}(j=1, \cdots, J)$. The normal vector of $P_{n i}$ is denoted by $N_{n i}$. A plane corresponding to the point $Q_{n i j}$ is searched from range data other than the range data $n$. A plane $P_{k l}$ corresponding to the point $Q_{n i j}$ is selected so that $\left|\overline{Q_{n i j} Q_{x}}\right|$, which means the distance between $Q_{n i j}$ and $Q_{x}$, is minimized. Note that $P_{k l}$ and $Q_{n i j}$ satisfy two conditions shown in Fig. 7: the inner product of $N_{k l}$ and $N_{n i}$ is below a threshold (Fig. 7(a)) and a point $Q_{x}$ where the vector $N_{k l}$ passing through point $Q_{n i j}$ intersects the plane $P_{k l}$ exists (Fig. 7(b)). $P_{k l 1}$ is chosen as the plane which 


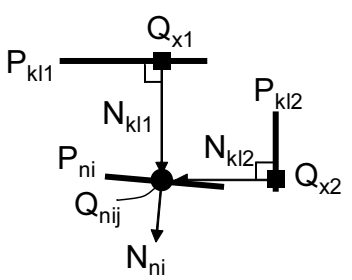

(a) Selection by threshold of an inner product.

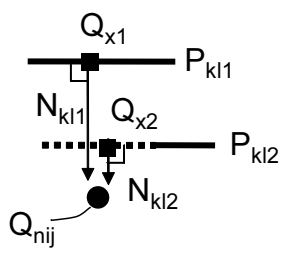

(b) Selection by existence of an intersection.

Figure 7. Selection of corresponding plane.

corresponding to $Q_{n i j}$ in both Fig. 7(a) and (b), .

\subsubsection{Estimation of position and orientation of range}

data. The sensor position of range data is estimated from the distance between a point and the corresponding plane and the sensor orientation of range data is estimated from the inner product of normal vectors of corresponding point and plane. Let $R_{n}$ and $T_{n}$ be sensor orientation and position of range data $n(n=1, \cdots, N)$, respectively.

step 1. The orientations $R_{n}$ are estimated by maximizing the correlation $C_{N}$ defined as the sum of inner products of normal vectors of a point $Q_{u}$ and a plane $P_{u}$ $(u=0, \cdots, U)$, where $Q_{u}$ is the point in $R D_{n 1}$ and $P_{u}$ is the plane in $R D_{n 2}, P_{u}$ is the plane which corresponds to the point $Q_{u}$, and $U$ represents the number of points which have corresponding planes.

$$
C_{N}=\sum_{u=0}^{U}\left(R_{n 1} N_{n u}\right) \cdot\left(R_{n 2} N_{m u}\right) \rightarrow \max ,
$$

where $N_{n u}$ and $N_{m u}$ are the normal vectors of $Q_{u}$ and $P_{u}$ respectively.

step 2. The positions $T_{n}$ are estimated by minimizing the error $E_{T}$ which is defined as the sum of distances between corresponding point and plane as follows.

$$
E_{T}=\sum_{u}^{U} \operatorname{distance}\left(Q_{u}^{\prime}, P_{u}^{\prime}\right) \rightarrow \min ,
$$

where $Q_{u}^{\prime}$ and $P_{u}^{\prime}$ are $Q_{u}$ and $P_{u}$ after transformation by $\left(R_{n 1}, T_{n 1}\right)$ and $\left(R_{n 2}, T_{n 2}\right)$, and the orientations estimated in step 1 are fixed.

The corresponding plane is searched again after the step 2 and the process is iterated until the solution is converged. Multiple dimension downhill simplex method [18], which does not need the derivatives, is used for the optimization.

\subsection{Polygonization of Range Data}

A polygonal representation is generated from each range data by connecting four corners of each region defined as a plane portion in the plane detection process in order to reduce the number of polygons. In a non-plane portion, a polygon is generated by connecting adjoining pixels which are its neighbors and one of its diagonal neighbors. A range data partially overlaps other range data. The quantity of data is reduced by removing redundant polygons at overlapping regions. Polygons are generated from range data in order of input. The generated polygons are searched which correspond to the vertices of the generating polygon with the method described in Section 3.1.2. When distances between vertices and intersection $Q_{x}$ are less than a threshold, the polygon is deleted as a redundant one as shown in Fig. 8 . Note that only the polygon defined as a plane portion is deleted to maintain the quality of model and to reduce the amount of data.

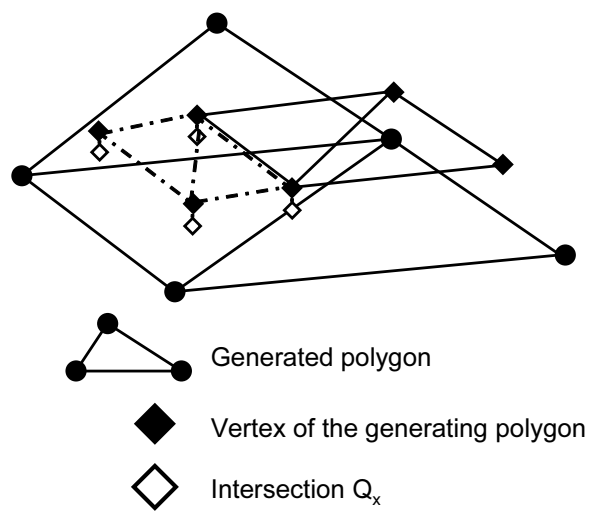

Figure 8. Deletion of overlapping areas.

\section{Generation of Textured Model}

The 3D shape obtained in the previous section is colored by textures selected from omnidirectional color images. The geometrical relationship between the rangefinder and the OMS is known by the alignment described in Section 2. Therefore, the camera position and orientation of omnidirectional images are also estimated by registering the range data, and the correspondence between a 2-D point in 
omnidirectional images and a 3-D point in range data can be easily determined.

\subsection{Selection of Omnidirectional Image}

Each polygon on the 3D shape is colored by the texture selected from the image which gives the highest resolution. A polygon is projected onto each omnidirectional image and then the image with the biggest area of the polygon is chosen. However, when occlusions occur this strategy fails. For example, as shown in Fig. 9, the resolution of texture generated from camera 2 is higher than the camera 1 . However, the correct texture can not be taken by the camera 2 . The occlusion is detected when the generated surface model intersects with a triangular pyramid determined by triangular patch vertices and the projection center of camera. In such a case, the next highest resolution image is selected, and occlusion is re-detected.

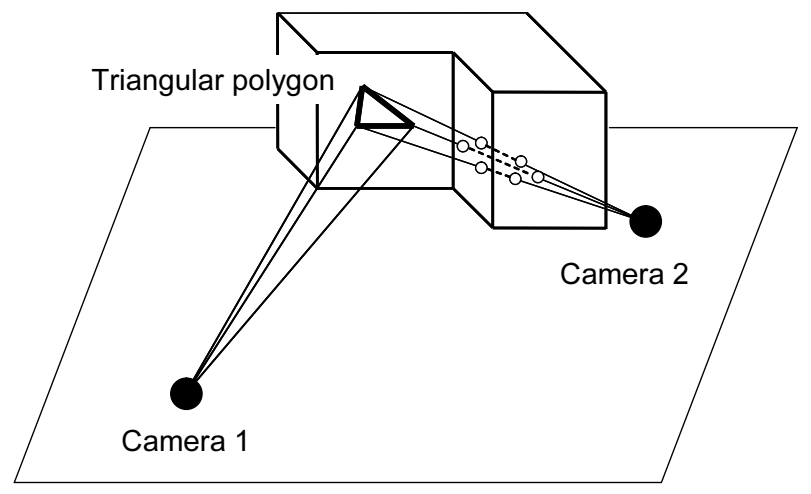

Figure 9. Selection of omnidirectional image in consideration of occlusion.

\section{Experiments}

We have carried out experiments of reconstructing our campus. In experiments, the range and color images are acquired at 68 points in our campus (about $250 \mathrm{~m} \times 300 \mathrm{~m}$ ). Fig. 10 illustrates the data acquisition points in our campus. Since the proposed method requires overlapping portions among range data for registration, we have acquired the data paying that attention. The sensor coordinate systems are aligned in advance by the method described in Section 2.2. The resolution of each omnidirectional range image is $1024 \times 512$. A cluster consisting of 24 PCs (CPU: Pentium4 1.7Ghz, Memory: 1024MB) is used for finding corresponding plane, and single PC (CPU: Pentium4 1.8Ghz, Memory: $2048 \mathrm{MB}$ ) is used for registration of multiple range data. The time required for registration is about 7 days. In registration, the range data acquired at point $\mathrm{A}$ in Fig. 10 is

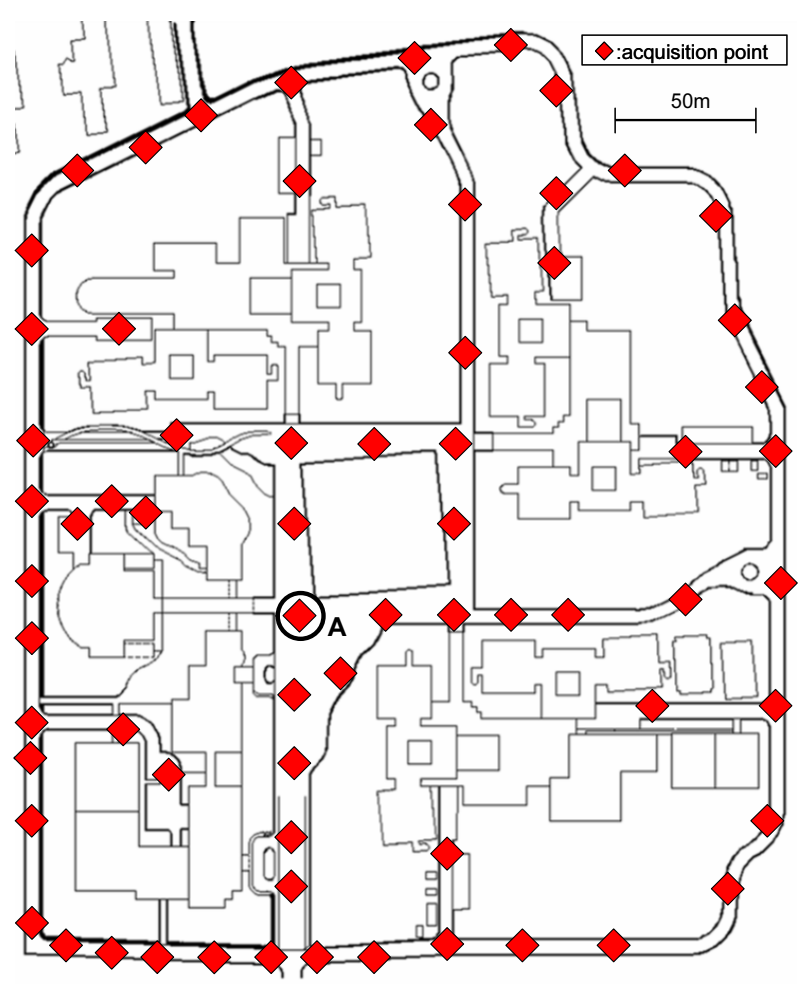

Figure 10. Range data acquisition points.

selected as a reference. Fig. 11 illustrates a 2D CAD data of our campus overlaid with the generated model. Examples of rendering the generated model are shown in Fig. 12.

\section{Conclusion}

This paper has proposed a 3D modeling method which is based on integrating omnidirectional range and color images for wide area outdoor environments. In experiments, a 3D model is actually generated from omnidirectional range and color images acquired at 68 points in our campus. We can observe many small data lacking portions mainly caused by occlusions in the generated model. In order to measure them efficiently, we are planning to carry out continuous measurement during the movement of sensors. Moreover, the sense of incongruity is observed in the generated model, when different images are selected in neighboring polygons. Such an effect is mainly caused by the varying illumination conditions during the measurement of the whole area. This problem in generating a textured 3D model should further be investigated.

\section{References}

[1] T. Sato, M. Kanbara, N. Yokoya and H. Takemura: "Dense 3-D Reconstruction of an Outdoor Scene by Hundredsbaseline Stereo Using a Hand-held Video Camera," Interna- 


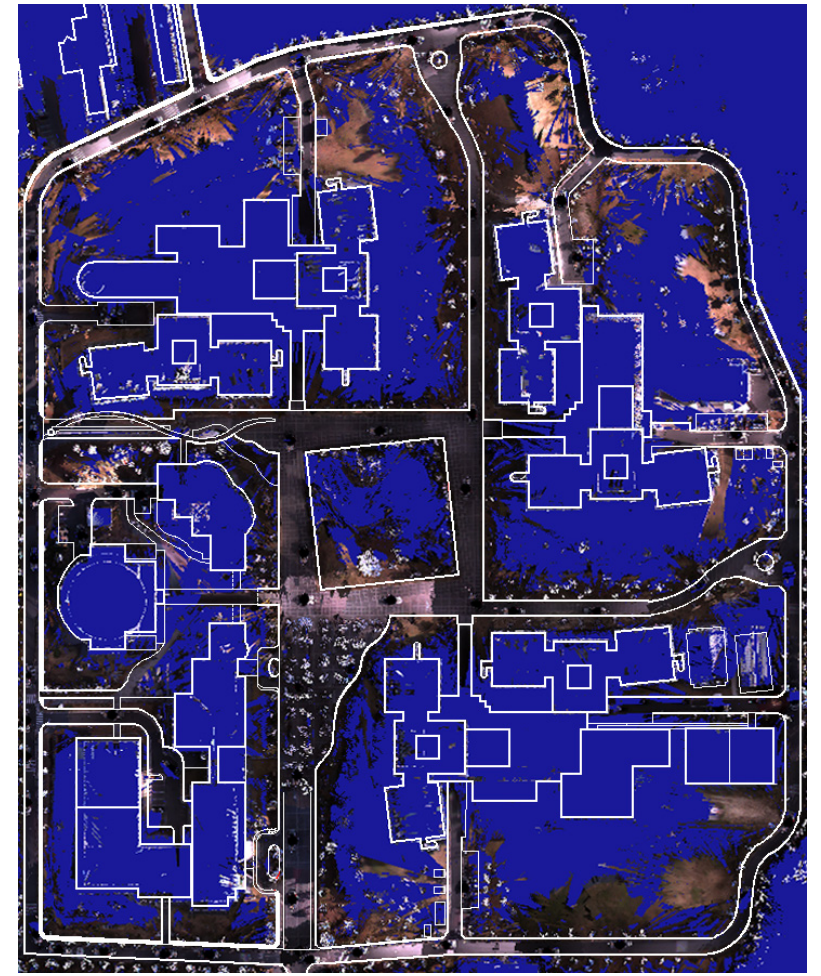

Figure 11. 2D CAD data overlaid on generated 3D model

tional Jour. of Computer Vision, Vol. 47, No. 1-3, pp. 119 129, 2002.

[2] S. Ozawa, M. Notomi, and H. Zen: "A Wide Scope Modeling to Reconstruct Urban Scene", Proc. ISPRS Commission V Int. Symposium on Real-Time Imaging and Dynamic Analysis, pp. 370-376, 1998.

[3] C. Tomasi and T. Kanade: "Shape and Motion from Image Streams under Orthography A Factorization Method," International Jour. of Computer Vision, Vol. 9, No. 2, pp. 137154, 1992.

[4] M. Pollefeys, R. Koch, M. Vergauwen, A. A. Deknuydt and L. J. V. Gool: "Three-demintional Scene Reconstruction from Images," Proc. SPIE, Vol. 3958, pp. 215-226, 2000.

[5] M. Okutomi and T. Kanade: "A Multiple-baseline Stereo," IEEE Trans. on Pattern Analysis and Machine Intelligence, Vol. 15, No. 4, pp. 353-363, 1993.

[6] S. F. El-Hakim, C. Brenner, and G. Roth: "A Multi-sensor Approach to Creating Accurate Virtual Environments," Jour. of Photogrammetry \& Remote Sensing, Vol. 53, pp. 379391, 1998.

[7] H. Zhao and R. Shibasaki: "Reconstruction of Textured Urban 3D Model by Fusing Ground-Based Laser Range and CCD Images," IEICE Trans. Inf. \& Syst., Vol. E-83-D, No. 7, pp. 1429-1440, 2000.

[8] H. Zhao and R. Shibasaki: "Reconstructing a Textured CAD
Model of an Urban Environment Using Vehicle-Borne Laser Range Scanners and Line Cameras," Machine Vision and Applications, Vol. 14, No. 1, pp. 35-41, 2003.

[9] P. K. Allen, A. Troccoli, B. Smith, S, Murray, I. Stamos, and M. Leordeanu: "New Methods for Digital Modeling of Historic Sites," IEEE Computer Graphics and Applications, Vol. 23, pp. 32-41, 2003.

[10] Y. Sun, J. K. Paik, A. Koschan and M. A. Abidi: "3D Reconstruction of Indoor and Outdoor Scenes Using a Mobile Range Scanner," Proc. Int. Conf. on Pattern Recognition, Vol. 3, pp. 653-656, 2002.

[11] C. R. Gunadi, H. Shimizu, K. Kodama, and K. Aizawa: "Construction of Large-scale Virtual Eenvironment by Fusing Range Data, Texture Images and Airbone Altimetry Data," Proc. Int. Symposium on 3D Data Processing, Visualization and Transmission, pp. 772-775, 2002.

[12] C. Früh and A. Zakhor: "Constructing 3D City Models by Merging Aerial and Ground Views," IEEE Computer Graphics and Applications, Vol. 23, pp. 52-61, 2003.

[13] Y. Kanazawa and K. Kanatani: "Reliability of Fitting a Plane to Range Data," IEICE Trans. on Information and Systems, Vol. E78-D, No. 12, pp. 1630-1635, 1995.

[14] S. Ikeda, T. Sato, and N. Yokoya: "Panoramic Movie Generation Using an Omnidirectional Mmulti-camera System for Telepresence", Proc. 13th Scandinavian Conf. on Image Analysis, pp. 1074-1081, 2003.

[15] P. J. Besl and N. D. McKay: "A Method for Registration of 3-D Shapes," IEEE Trans. on Pattern Analysis and Machine Intelligence, Vol. 14 No. 2, pp. 239-256, 1992.

[16] K. Pulli: "Multiview Registration for Large Data Sets," Proc. Int. Conf. on 3D Digital Imaging and Modelling, pp. 160$168,1999$.

[17] T. Oishi, R. Sagawa, A. Nakazawa, R. Kurazume, and K. Ikeuchi: "Parallel Alignment of a Large Number of Range Images," Proc. Int. Conf. on 3D Digital Imaging and Modeling, pp. 195-202, 2003.

[18] W. H. Press, B. P. Flannery, S. A. Teukolsky, and W. T. Vetterling: Numerical Recipes in C, Cambridg University Press 1988. 


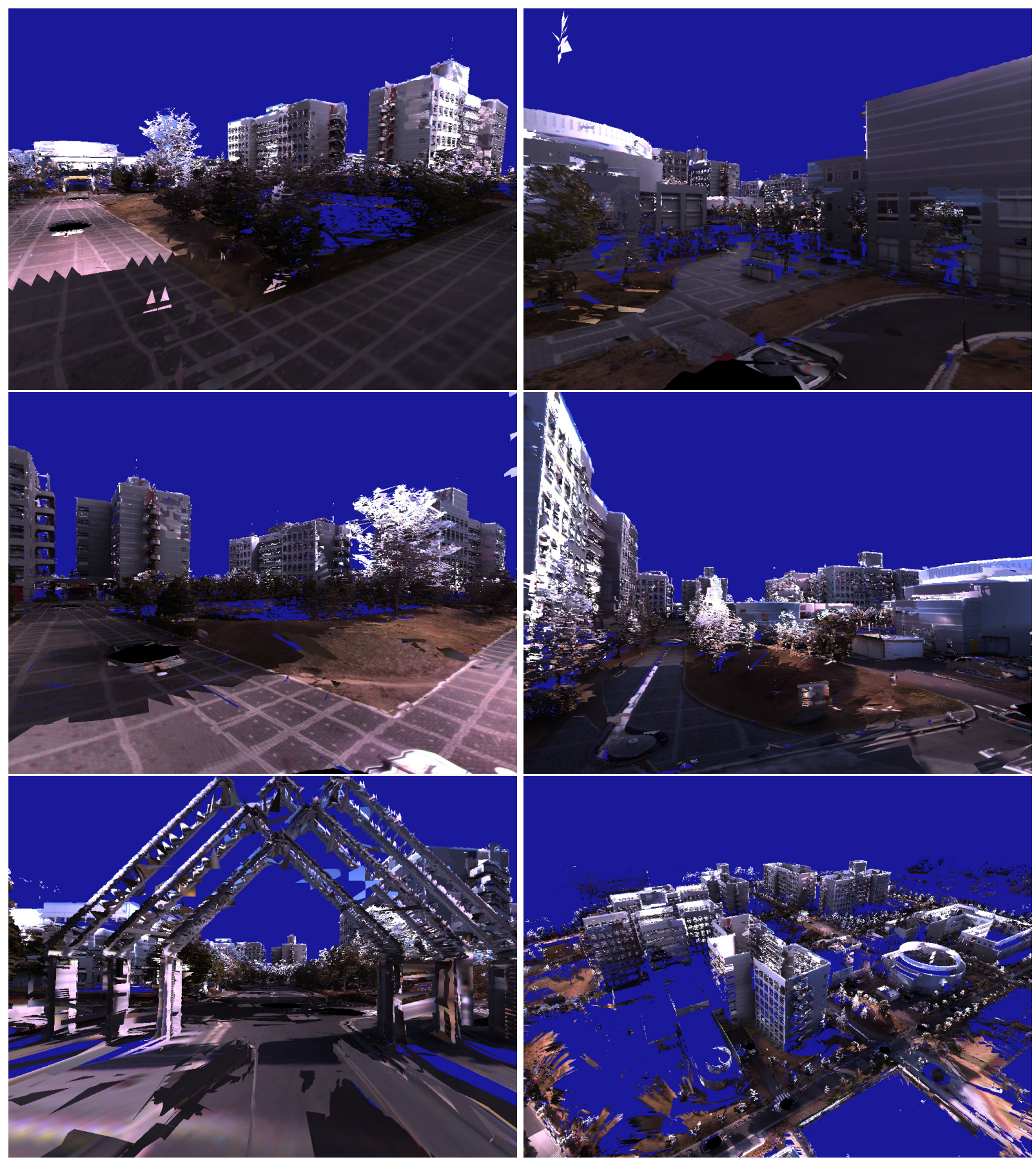

Figure 12. Generated 3D model with texture 